\title{
Structural, magnetic and low-temperature electrical transport properties of YIG thin films with heavily reduced oxygen contents
}

Venkat Swamy ${ }^{\dagger}$ Shu Mi`, Haoliang Huang, Chenguang Mei, Yalin Lu, Dongsheng Song, Haifeng Du and Yonggang Zhao*

Dr. Venkat Swamy†, Dr. Shu Mi†, Chenguang Mei, Prof. Yonggang Zhao

Department of Physics and State Key Laboratory of Low-Dimensional

Quantum Physics, Tsinghua University, Beijing 100084, China

Frontier Science Center for Quantum Information, Tsinghua University, Beijing 100084, China

Haoliang Huang, Prof. Yalin Lu

Hefei National Laboratory for Physical Sciences at the Microscale \& National Synchrotron Radiation Laboratory, University of Science and Technology of China, Hefei 230026, China

Prof. Dongsheng Song

Institutes of Physical Science and Information Technology, Anhui University, Hefei 230601, China

Prof. Haifeng Du

Anhui Province Key Laboratory of Condensed Matter Physics at Extreme Conditions, High Magnetic Field Laboratory of Chinese Academy of Sciences, and University of Science and Technology of China, Hefei, 230031, China 


\section{Supporting Information}

*E-mail: ygzhao@tsinghua.edu.cn

†These authors contributed equally to this work.

\section{S1 XRD patterns of the different YIG thin films}
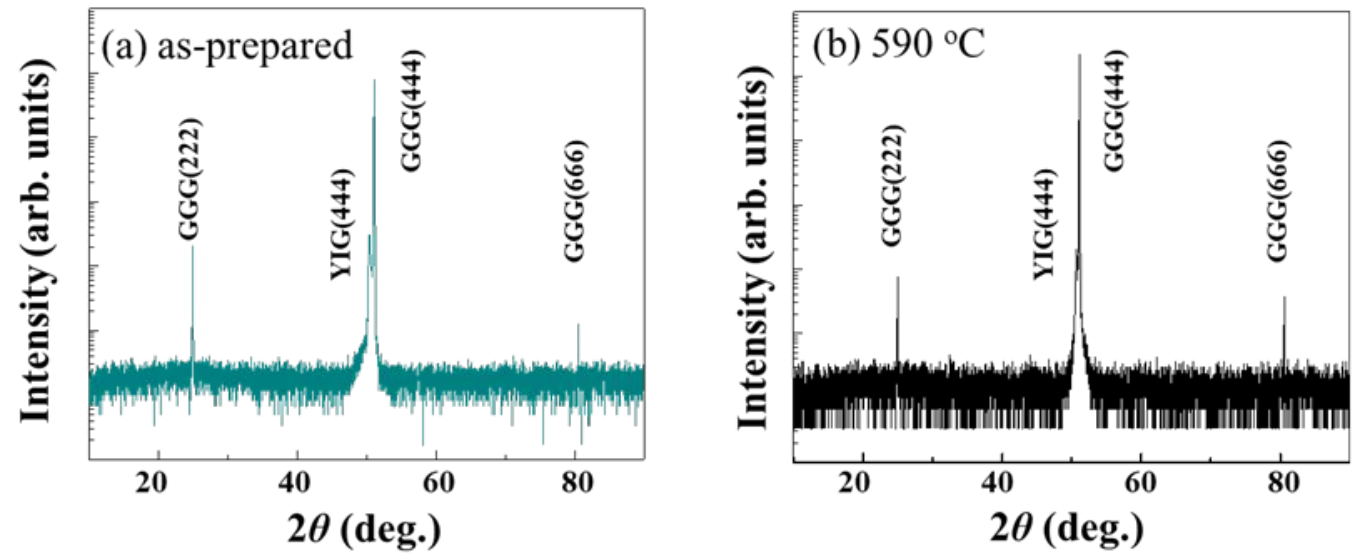

Figure S1. XRD patterns of $2 \theta$ from 10-90 degree of (a) the as-prepared and (b) 590 ${ }^{\circ} \mathrm{C}$ annealed films.

Figure S1 shows the XRD patterns of $2 \theta$ from 10-90 degree of the as-prepared and $590{ }^{\circ} \mathrm{C}$-annealed films. The comparison between the two patterns indicates that only YIG peaks are present in the annealed sample and no other peaks related to the impurity phase can be seen. This means that the YIG films still maintain the lattice structure after annealing.

\section{S2 TEM images of the different YIG thin films}




\section{Supporting Information}

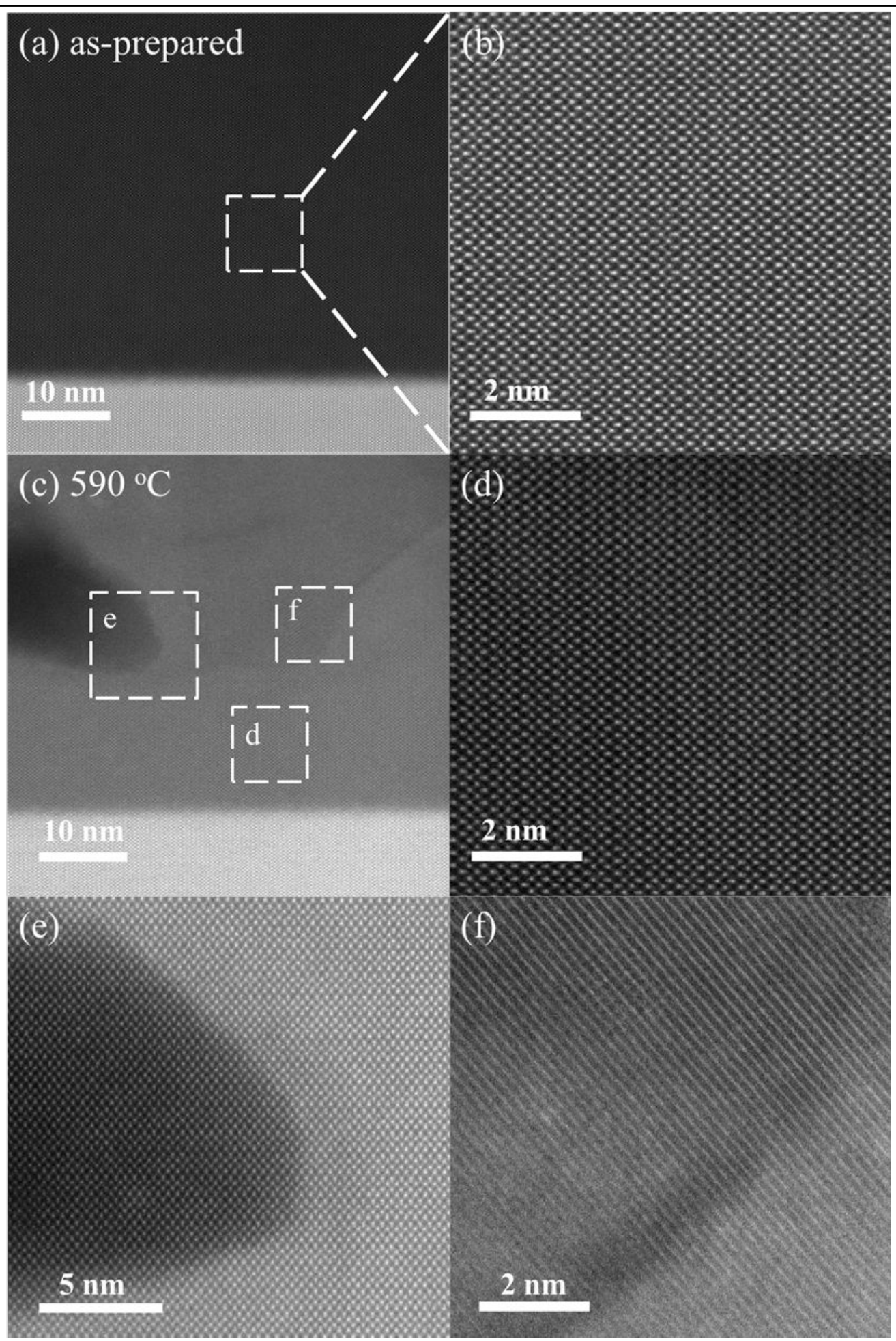

Figure S2. TEM results: (a) Low magnification and (b) high magnification cross-section view for the as-prepared films. (c) Low magnification cross-section view for the $590{ }^{\circ} \mathrm{C}$ annealed films. (d)-(f) Magnified regions of panel $\mathrm{d}$ corresponding to the areas of $\mathrm{d}$, e and $\mathrm{f}$. 


\section{Supporting Information}

In order to confirm the YIG lattice structure, the cross-section TEM images of the as-prepared and $590^{\circ} \mathrm{C}$ annealed YIG films are presented in Figure S2. It is found that the as-prepared film shows homogeneous crystalline quality (Figure S2a). The high resolution image (Figure S2b) shows obvious YIG film lattices. The composition of the as-prepared films was measured by energy dispersive spectrum (EDS). The ratio of $\mathrm{Y}: \mathrm{Fe}: \mathrm{O}$ elements is $3: 4.7: 10$, which is roughly consistent with the stoichiometry of YIG in the experimental error ranges (the ratio of Y:Fe:O elements is 3:5:12). Figure S2c shows the TEM image of the $590{ }^{\circ} \mathrm{C}$ annealed films. It is found that the color of image is not homogeneous. The high resolution image of the homogeneously area (Figure S2d) shows a good YIG lattice, which indicates that the YIG lattice remains after annealing. In this area, the ratio of $\mathrm{Y}: \mathrm{Fe}: \mathrm{O}$ elements is 3:5.1:8.1. The oxygen content is lower than that of the as-prepared films, and the ratio of $\mathrm{Y}: \mathrm{Fe}$ is almost consistent with that of the as-prepared films. Figure S2e,f shows the high resolution image of the inhomogeneous areas. It was found that the lattice structure is still continuous, indicating that this areas are still YIG lattice. In the dark area of Figure S2e, the ratio of $\mathrm{Y}: \mathrm{Fe}: \mathrm{O}$ is $3: 4.9: 6.2$, indicating that the oxygen content is lower than that of the bright areas (8.1). It means the inhomogeneous of color may be due to the inhomogeneous distribution of oxygen content in the YIG films, which can account for the increase of the XRD peak FWHM after annealing (Figure 1a in the main text).

S3 XRD patterns of the vacuum-annealed and graphite-annealed YIG thin films 


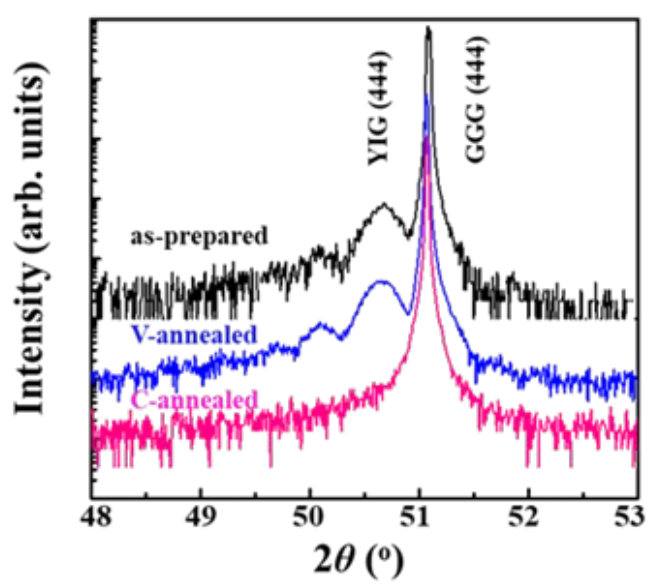

Figure S3. XRD patterns of the as-prepared, vacuum-annealed and graphite-annealed YIG thin films at $600{ }^{\circ} \mathrm{C}$.

In order to determine whether graphite-annealing has a significant effect on the structural of YIG films, XRD patterns of the as-prepared, vacuum-annealed (V-annealed) and graphite-annealed (C-annealed) samples at $600{ }^{\circ} \mathrm{C}$ were measured. As shown in Figure S3, there is no change in the YIG (444) peaks before and after V-annealed at $600{ }^{\circ} \mathrm{C}$. However, after C-annealed at $600{ }^{\circ} \mathrm{C}$, the YIG (444) peak disappears. As mentioned in the main text (Figure 1a), the YIG (444) peak shifts to the higher angles with decreased intensities, it is likely that the YIG (444) peak overlaps with the GGG (444) peak. Therefore, we can deduce that compared with V-annealing, which does not show obvious effect, graphite-annealing can effectively change the structure of YIG.

\section{S4 $M-H$ and $\rho-T$ curves of re-annealed YIG thin films}



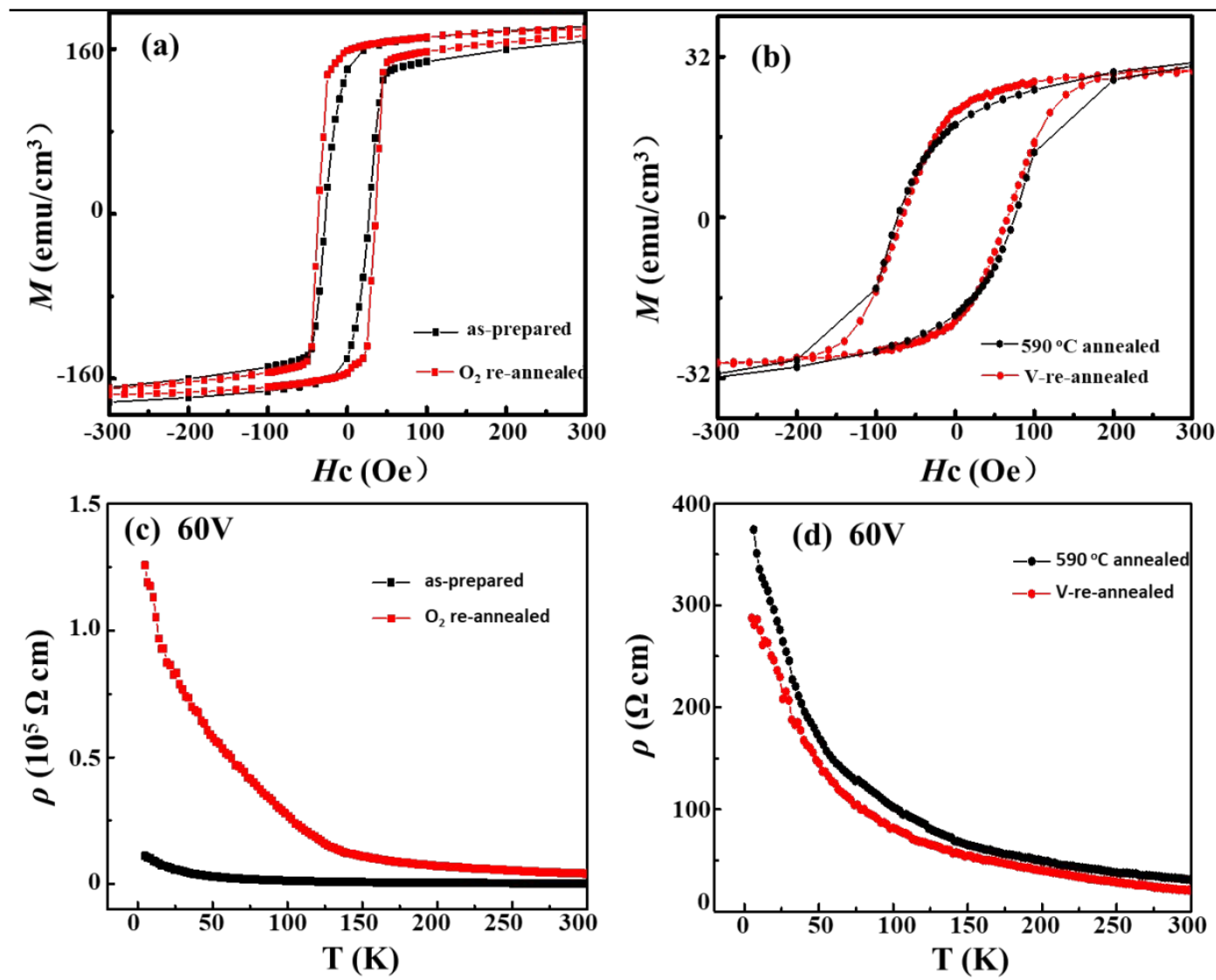

Figure S4. (a,b) $M-H$ curves and (c, d) $\rho$ - $T$ curves under $60 \mathrm{~V}$ for the as-prepared, 590 ${ }^{\circ} \mathrm{C}$ (carbon) annealed, oxygen $\left(\mathrm{O}_{2}\right.$ re-annealed $)$ and vacuum re-annealed (V-re-annealed) of the $590{ }^{\circ} \mathrm{C}$ carbon-annealed films.

The magnetic hysteresis $(M-H)$ loops of the oxygen and vacuum re-annealed YIG films were measured, as shown in Figure S4a,b. It can be found that the $M-H$ loop of YIG films does not show obvious change after vacuum re-annealing (compared to that of the $590{ }^{\circ} \mathrm{C}$ carbon-annealed film). But after oxygen re-annealing, the $M \mathrm{~s}$ increases to $180 \mathrm{emu} / \mathrm{cm}^{3}$ and $H \mathrm{c}$ is decreased to $35 \mathrm{Oe}$, which is in agreement with that of the as-prepared films. These results suggest that carbon annealing only leads to the reduction of oxygen contents and results in the change of magnetic property. 


\section{Supporting Information}

Figure S4c,d shows the $\rho$-T curves of oxygen and vacuum re-annealed YIG thin films. After vacuum re-annealing, the $\rho$-T curve does not show obvious change. However, the oxygen re-annealing leads to the obvious increase in resistivity which is even an order of magnitude higher than that of as-prepared films at low temperatures. This resistivity difference existed even for the as-prepared films. Because the resistivity for the as-prepared films is very sensitive to the oxygen content, a slight variation of oxygen content will lead to a large change of resistivity.

\section{S5 XAS results of different YIG thin films}

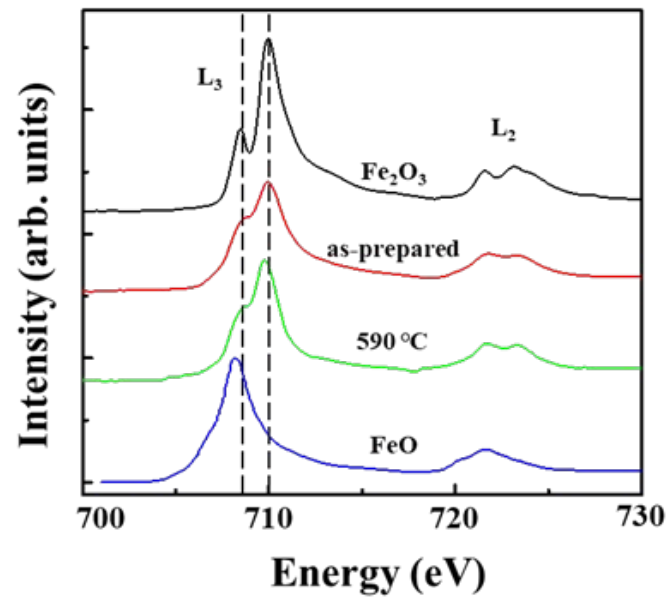

Figure S5. The Fe $\mathrm{L}_{2}$ and $\mathrm{L}_{3} \mathrm{XAS}$ spectra of the as-prepared film, $590{ }^{\circ} \mathrm{C}$-annealed YIG film, and standard samples of $\mathrm{Fe}_{2} \mathrm{O}_{3}\left(\mathrm{Fe}^{3+}\right)$ and $\mathrm{FeO}\left(\mathrm{Fe}^{2+}\right)$.

Figure S5 shows the $\mathrm{Fe} \mathrm{L}_{2}$ and $\mathrm{L}_{3} \mathrm{XAS}$ spectra of the as-prepared and 590 ${ }^{\circ} \mathrm{C}$-annealed YIG films. Compared with the standard samples $\mathrm{Fe}_{2} \mathrm{O}_{3}\left(\mathrm{Fe}^{3+}\right)$ and $\mathrm{FeO}$ $\left(\mathrm{Fe}^{2+}\right)$, it can be clearly seen that the peak shape of spectra of both samples is close to that of $\mathrm{Fe}_{2} \mathrm{O}_{3}$. This means $\mathrm{Fe}$ is mainly trivalent in both samples. Moreover, for the $590{ }^{\circ} \mathrm{C}$-annealed samples, the $\mathrm{L}_{3}$ main peak shifts to lower energy, indicating that a 


\section{Supporting Information}

small amount of $\mathrm{Fe}^{2+}$ was created.

\section{S6 Estimation of magnetization change based on the Fe valence change}

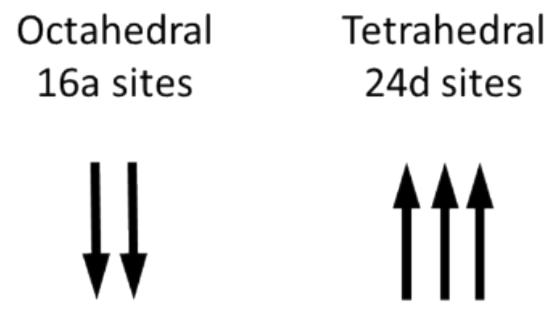

Figure S6. Magnetic moment orientation of the Fe ions in YIG.

For YIG, as shown in Figure S6, 2/5 of total Fe ions are on the octahedral (16a) sites and $3 / 5$ of total Fe ions on the tetrahedral (24d) sites. ${ }^{[1]}$ The magnetic moments of Fe ions on the two sites are coupled antiferromagnetically. According to the XPS results (Figure 1c in the main text), the Fe ions is trivalent with $5 \mu_{\mathrm{B}}$ in the as-prepared films, which leads to the saturation magnetization $M_{s}=n(3 / 5-2 / 5) \times 5 \mu_{\mathrm{B}}=n \mu_{\mathrm{B}}$, where $\mathrm{n}$ is the number of $\mathrm{Fe}$ ions per volume. After $590{ }^{\circ} \mathrm{C}$ annealing, $35 \% \mathrm{Fe}^{3+}$ ions transform to $\mathrm{Fe}^{2+}$ ions with $4 \mu_{\mathrm{B}}$. If $\mathrm{Fe}^{2+}$ ions equally distribute at $16 \mathrm{a}$ and $24 \mathrm{~d}$ site, the contribution of $\mathrm{Fe}^{3+}$ ions to $M_{\mathrm{s}}$ is $M_{s}\left(\mathrm{Fe}^{3+}\right)=65 \% n \mu_{\mathrm{B}}$. While, the contribution of $\mathrm{Fe}^{2+}$ ions to the $M_{\mathrm{s}}$ is $M_{s}\left(F e^{2+}\right)=35 \% \times n(3 / 5-2 / 5) \times 4 \mu_{\mathrm{B}}=28 \% n \mu_{\mathrm{B}}$. The total $M_{s}=M_{s}\left(F e^{3+}\right)+M_{s}\left(F e^{2+}\right)=93 \% n \mu_{\mathrm{B}}$, which is $7 \%$ lower than the as-prepared films. If most of $\mathrm{Fe}^{2+}$ ions at $24 \mathrm{~d}$ sites, the contribution of $\mathrm{Fe}^{3+}$ ions to $M_{\mathrm{s}}$ is $M_{s}\left(\mathrm{Fe}^{3+}\right)=n[(3 / 5-35 \%)-2 / 5] \times 5 \mu_{\mathrm{B}}=-75 \% n \mu_{\mathrm{B}}$, and the contribution of $\mathrm{Fe}^{2+}$ ions to $M_{\mathrm{s}}$ is $M_{s}\left(F e^{2+}\right)=35 \% n \times 4 \mu_{B}=140 \% n \mu_{B}$. So the total 


\section{Supporting Information}

$M_{s}=M_{s}\left(F e^{3+}\right)+M_{s}\left(F e^{2+}\right)=65 \% n \mu_{\mathrm{B}}$, which is $35 \%$ lower than the as-prepared films.

\section{S7 $M-T$ results of different YIG thin films}

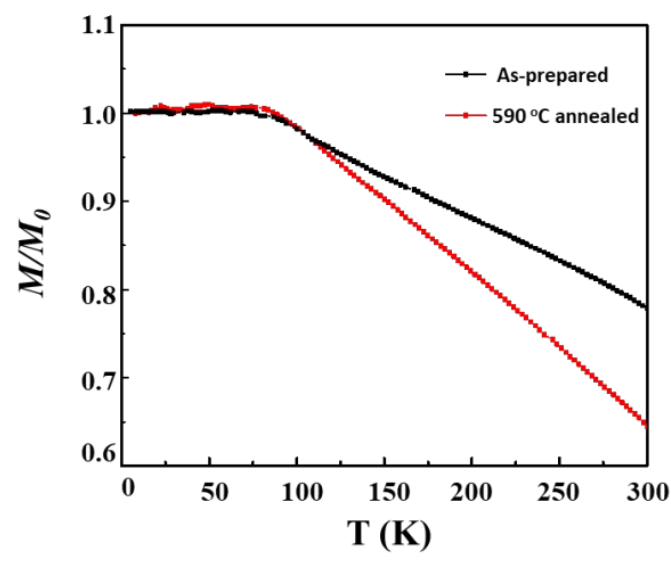

Figure S7. $M-T$ curves of the as-prepared and $590^{\circ} \mathrm{C}$-annealed films, $H=50$ Oe.

Figure S7 shows the $M-T$ curves of the as-prepared and $590{ }^{\circ} \mathrm{C}$-annealed films.

The measurement error is relatively large at low temperatures $(<40 \mathrm{~K})$ due to the strong paramagnetic signal from GGG substrate which was subtracted. In order to get information on the change of magnetic order temperature, the magnetization was normalized. It can be seen that the magnetizations for both samples increase monotonically with decreasing temperature and reach the saturation value at low temperatures. This behavior is consistent with that of the previous reports, ${ }^{[2,3]}$ as shown in Figure R7b. The results suggest that the magnetic order remains after annealing. However, the slope of $M-T$ curve for the $590{ }^{\circ} \mathrm{C}$-annealed film is lower than that of the as-prepared films, implying that the Curie temperature $(T \mathrm{c})$ decreases with the reduction of oxygen content, which is consistent with the previous reports. ${ }^{[4]}$ This can be attributed to the weakening of the exchange interaction and the resultant 


\section{Supporting Information}

decrease of $T \mathrm{c}$ due to oxygen content reduction.

\section{S8 FMR results of different YIG thin films}
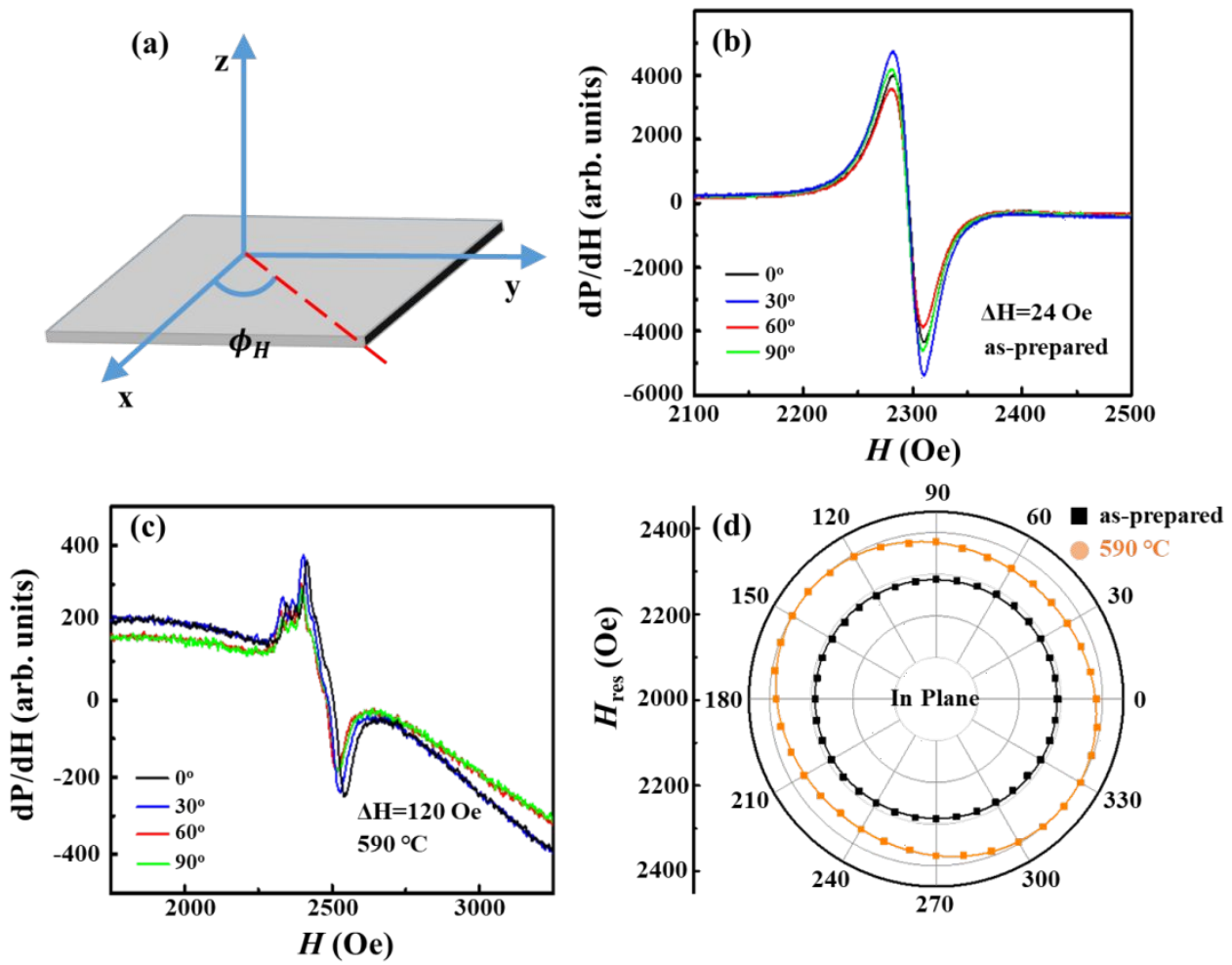

Figure S8. (a) Schematic of the in-plane FMR measurements. The in-plane FMR spectra of (b) the as-prepared and (c) $590{ }^{\circ} \mathrm{C}$-annealed YIG films. (d) Angular dependence ( $\left.\phi_{H}\right)$ of the resonance field $\left(H_{\text {res }}\right)$ for the YIG films.

Figure S8a shows the schematic of the in-plane FMR measurements. The magnetic field $H$ is rotated at an angle $\phi_{\mathrm{H}}$ in the plane. Figure S8b,c shows four representative FMR spectra of the as-prepared and $590{ }^{\circ} \mathrm{C}$-annealed YIG films at $\theta_{\mathrm{H}}=$ $0^{\circ}, 30^{\circ}, 60^{\circ}$, and $90^{\circ}$, respectively. It is found that there is no anisotropy in the as-prepared film. However, the annealed sample shows a weak in-plane anisotropy. In addition, the FMR linewidth $(\Delta H)$ increases from 24 to 120 Oe after the sample was 


\section{Supporting Information}

annealed. This may be attributed to the degradation of crystalline quality of YIG thin

films induced by oxygen vacancies.
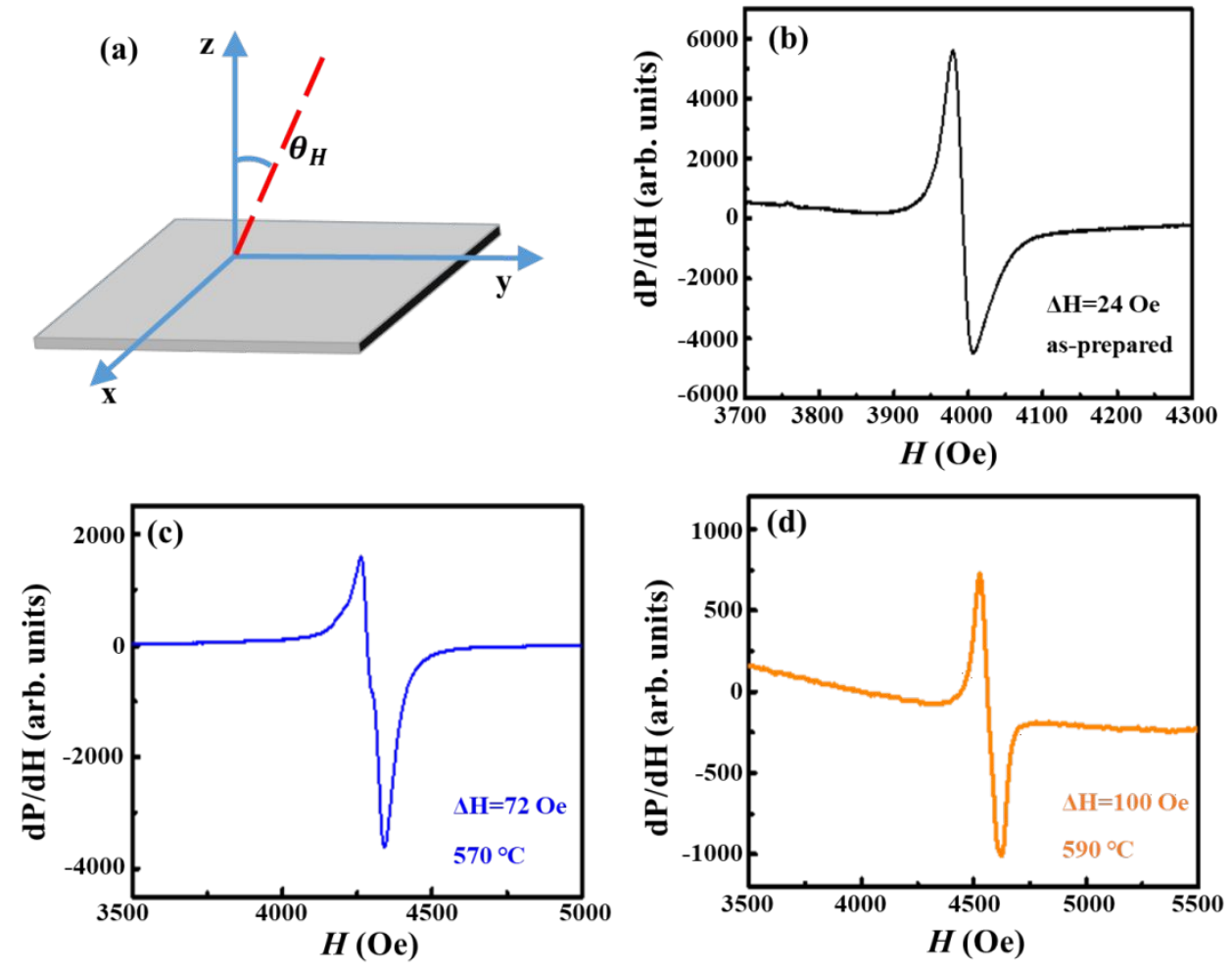

Figure S9. (a) Schematic of the out-of-plane FMR measurements. The out-of-plane

FMR spectra of (b) as-prepared, (c) $570{ }^{\circ} \mathrm{C}$ and (d) $590{ }^{\circ} \mathrm{C}$-annealed YIG films.

Figure S9a shows the schematic of the out-of-plane FMR measurements. The magnetic field $H$ is applied at an angle $\theta_{\mathrm{H}}$ with respect to the film normal. Figure S9b-d shows the representative out-of-plane FMR spectra of the as-prepared, $570^{\circ} \mathrm{C}$ and $590{ }^{\circ} \mathrm{C}$-annealed YIG films, respectively. It is found that the FMR linewidth $(\Delta H)$ increases with the annealing temperature increase, which is consistent with that of the in-plane results. 


\section{Supporting Information}

\section{S9 Estimation of Joule heating in measurement of $\rho$ - $T$ curves}

The temperature increase $\Delta T$ of the film at the temperature $T$ induced by overheating can be evaluated as a function of the applied electric field E by the expression of $\Delta T(T, E) \approx 2 P_{l} / \kappa_{\text {sub }}=2 E^{2} S\left[\rho(T+\Delta T) \kappa_{\text {sub }}\right]^{-1}$, where $P_{1}$ is the power dissipated per unit length of the sample, $\kappa_{\text {sub }}$ is the thermal conductivity of the substrate, $S$ is the cross section of the film, and $\rho$ is the resistivity of the sample. ${ }^{[5]}$ Using the experimental values of the resistivity at different temperatures, the electric fields, cross section of the film and the average value of $\kappa_{\text {sub }}=15 \mathrm{Wm}^{-1} \mathrm{~K}^{-1}$ in the temperature range $10-170 \mathrm{~K},{ }^{[5]}$ it was found that at $100 \mathrm{~K}$ the maximum increase of temperature due to overheating is about $0.1 \mathrm{~K}$, and at $10 \mathrm{~K}$, the maximum increase of temperature is about $0.001 \mathrm{~K}$. Therefore, Joule heating is a minor effect in our measurement and the observed ER is an intrinsic effect.

\section{S10 AFM results of different YIG thin films}

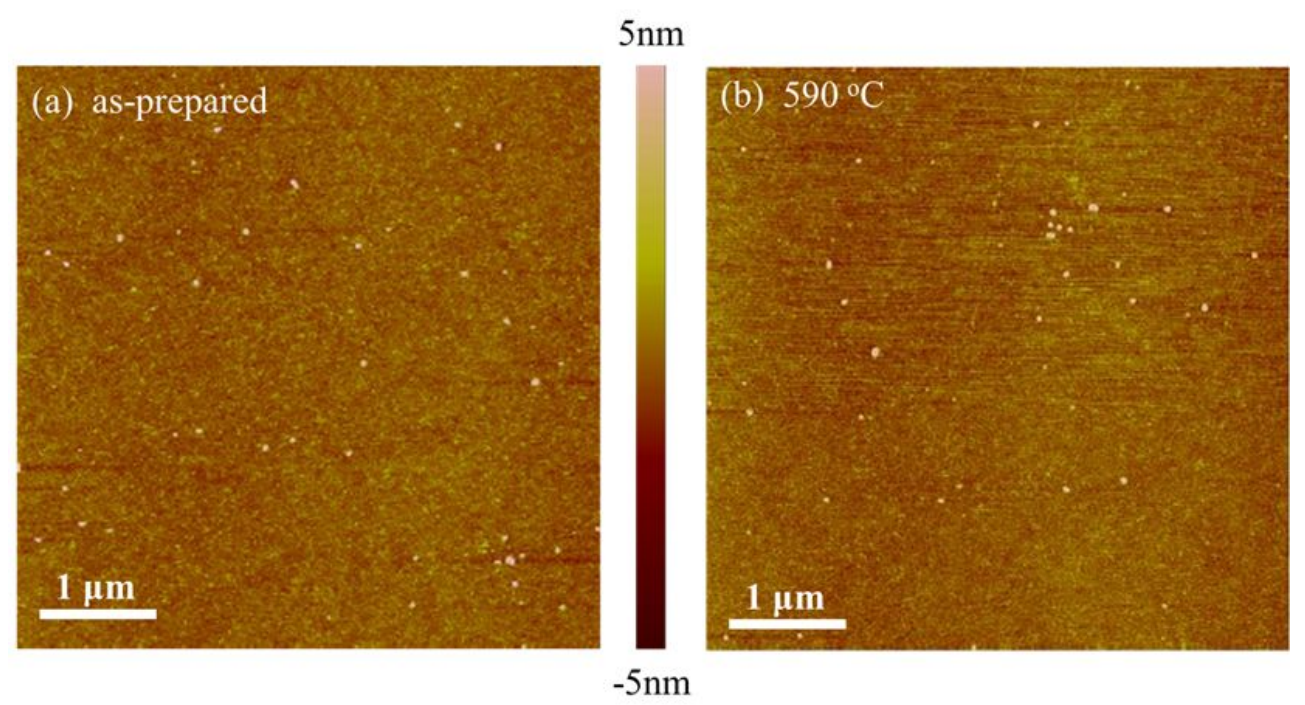

Figure S10. AFM images of (a) the as-prepared and (b) $590^{\circ} \mathrm{C}$ annealed films. 


\section{Supporting Information}

The surface morphology of YIG films was examined by atomic force microscopy (AFM), as shown in Figure S10. The surface of the as-prepared film is very smooth with a root-mean-square (RMS) roughness of $0.54 \mathrm{~nm}$ (Figure S10a). For the $590{ }^{\circ} \mathrm{C}$-annealed sample, the RMS roughness is $0.61 \mathrm{~nm}$ (Figure S10b), indicating that the morphology of the films does not change significantly after annealing.

\section{References}

[1] Gilleo, M. A.; Geller, S. Magnetic and Crystallographic Properties of Substituted Yttrium-Iron Garnet, $3 \mathrm{Y}_{2} \mathrm{O}_{3} \cdot \mathrm{xM}_{2} \mathrm{O}_{3} \cdot(5-\mathrm{x}) \mathrm{Fe}_{2} \mathrm{O}_{3}$. Phys. Rev. 1958, 110,73 .

[2] Anderson, E. E. Molecular Field Model and the Magnetization of YIG. Phys. Rev. 1964, 134, 1581

[3] Geller, S. Magnetic Study of the Lighter Rare-Earth Ions in the Iron Garnets. Phys. Rev. 1963, 131, 1080

[4] Dumont, Y.; Keller, N.; Popova, E.; Schmool, D. S.; Tessier, M.; Bhattacharya, S.; Stahl, B.; Da Silva, R. M. C.; Guyot, M. Tuning magnetic properties with off-stoichiometry in oxide thin films: An experiment with yttrium iron garnet as a model system. Phys. Rev. B. 2007, 76, 104413.

[5] Lavrov, A. N.; Tsukada, I.; Ando, Y. Normal-state conductivity in underdoped $\mathrm{La}_{2-\mathrm{x}} \mathrm{Sr}_{\mathrm{x}} \mathrm{CuO}_{4}$ thin films: Search for nonlinear effects related to collective stripe motion. Phys. Rev. B. 2003, 68, 094506. 